\title{
Sistemas Globais de Insumo-Produto: Uma Comparação entre o ICIO e a WIOT
}

\author{
Carlos Alberto Gonçalves Júnior ${ }^{1}$ \\ ${ }^{1}$ Departamento de Economia da Universidade Estadual do Oeste do Paraná. E-mail: carlosalbertojr@hotmail.com
}

\begin{abstract}
RESUMO
O presente artigo tem como objetivo comparar os sistemas globais de insumo produto Intercountry Input-Output Model ICIO da Organização para Cooperação e Desenvolvimento Econômico OCDE e a World Input-Output Table WIOT, desenvolvida pela World Input-Output Database WIOD, no intuito de identificar as principais diferenças existentes entre eles em termos holísticos e partitivos. Posteriormente, avaliou-se se a escolha entre os referidos sistemas pode influenciar os resultados da análise de insumo-produto. Os resultados mostraram que a aparentemente baixa proximidade, em termos partitivos, entre os valores estimados pelo ICIO e pela WIOT não resultou em maiores diferenças holísticas, isto é, não comprometeu os resultados da análise de insumoproduto para a grande maioria dos países. Todavia, quando se trata de uma análise específica para alguns países, como Malta, Chipre, Lituânia e Luxemburgo, e/ou alguns setores, principalmente os de serviços, alguns apontamentos feitos neste estudo precisam ser considerados.
\end{abstract}

\section{PALAVRAS-CHAVE}

Diferenças partitivas, Diferenças holísticas, Sistemas globais de insumo-produto

\section{Global Input-Output Systems: A Comparison between ICIO and WIOT}

\begin{abstract}
This article aims to compare the global input-output interregional systems Intercountry Input-Output Model ICIO from Organization for Economic Co-operation and Development OECD and World Input-Output Table WIOT from World Input-Output Database WIOD, in order to identify the main differences between them, in holistic and partitive terms. Subsequently, it was assessed whether the choice between these systems can influence the results of the input-output analysis. The results showed that the apparently low partitive proximity between the values estimated by ICIO and WIOT did not convert into greater holistic differences, i.e., it did not compromise the results of input-output analysis for the majority of countries. However, when it comes to a specific analysis for some countries, such as Malta, Cyprus, Lithuania and Luxembourg, and/or some industries, mainly services sectors, some notes made in this study need to be considered.
\end{abstract}

\section{KEYWORDS}

Partitive accuracy, Holistic accuracy, Global input-output systems

CLASSIFICAÇÃO JEL

C67, D57, R15 


\section{Introdução}

As matrizes insumo-produto nacionais têm sido amplamente utilizadas pelos planejadores da economia como uma ferramenta efetiva para analisar várias questões sociais e ambientais. Não obstante, a abordagem convencional, baseada em uma única perspectiva regional, tornou-se menos efetiva nos anos recentes devido às notáveis mudanças nas redes de produção, principalmente no que tange à fragmentação internacional dos processos produtivos e ao consequente aumento nas transações intermediárias, não só entre países vizinhos, mas também entre blocos inter-regionais, o que aumenta a relevância de sistemas globais de insumo-produto (Yamano, 2017).

Para Miller e Blair (2009), nos sistemas globais (ou internacionais) de insumoproduto, a estrutura segue o mesmo raciocínio dos modelos inter-regionais subnacionais. Em alguns casos, a coleta de dados torna-se até mais fácil, pois, enquanto os dados referentes às importações e exportações regionais (considerando regiões dentro de um mesmo país) são, na maioria das vezes, incompletos ou inexistentes, os mesmos dados podem ser coletados para grande parte dos países, considerando exportações ou importações para o exterior.

Contudo, quando se trata de modelos internacionais, outros problemas precisam ser superados, como: (i) problemas de transbordo, (ii) reexportação, (iii) reimportação e (iv) diferenças na valoração Cost, Insurance and Freight CIF e Free on Board FOB. Além disso, as matrizes de insumo-produto nacionais estão, em sua maioria, em preços básicos e os dados de comércio, disponibilizados nas bases internacionais, em preços ao consumidor. A forma como cada organização internacional, que se propõe a estimar os sistemas internacionais de insumo-produto, lida com esses problemas pode acarretar diferenças significativas nos sistemas estimados, o que pode levar a diferentes resultados.

Entre as principais bases de dados disponibilizadas para sistemas Globais Multirregionais baseados em modelos de insumo-produto estão o Intercountry Input-Output Model ICIO da Organização para Cooperação e Desenvolvimento Econômico OCDE Yamano (2017) e a Word Input-Output Data base WIOD Dietzenbacher et al. (2013). Além das bases EORA Lenzen et al. (2013); EXIOBASE Tukker e Dietzenbacher (2013) e Global Trade Analysis Project GTAP.

Outro exemplo interessante de modelo internacional de insumo-produto é o do Instituto de Desenvolvimento Econômico do Japão IDE-JETRO Meng et al. (2013) que produziu uma longa série de tempo de matrizes insumo-produto para os países asiáticos.

Nesse contexto, considerando-se a diversidade de sistemas multirregionais globais, identificar como a escolha entre os sistemas internacionais, publicados por várias organizações, pode afetar os resultados da análise de insumo-produto torna-se de suma importância e já é tema recorrente na literatura. 
A comparação entre sistemas multirregionais globais de insumo-produto já é objeto de vários trabalhos internacionais como o de Owen et al. (2014) que comparam os sistemas estimados por três organizações, Eora, GTAP e WIOD. Também Steen-Olsen et al. (2014) que compararam os resultados obtidos utilizando Eora, EXIOBASE, GTAP e WIOD no que diz respeito à sensibilidade dos multiplicadores de emissões de CO2 a diferentes agregações. Stadler et al. (2014) analisaram o comportamento da região chamada de "Restante do Mundo" entre a EXIOBASE, o GTAP e a WIOD. Moran e Wood (2014) também analisaram a convergência entre as bases Eora, WIOD, EXIOBASE e GTAP em relação à emissão de carbono.

Pode-se observar que a literatura anteriormente citada não utiliza em suas comparações o Intercountry Input-Output ICIO, desenvolvido pela Organização para Cooperação e Desenvolvimento Econômico. Além disso, a World Input-Output Table WIOT, desenvolvida pela World Input-Output Database WIOD está presente em todas as análises. Nesse contexto, o presente trabalho tem como objetivo comparar os sistemas inter-regionais ICIO e WIOD no intuito de observar suas diferenças, em termos partitivos e holísticos, no intuito de contribuir para a literatura já existente.

No presente artigo, comparam-se os sistemas internacionais estimados pela WIOT e pelo ICIO nos anos de 1995 e 2011. Os referidos períodos foram escolhidos por serem os anos extremos das séries disponíveis nas publicações compativeis International Standard Industrial Classification ISIC 3, tanto para o ICIO quanto para a WIOT. Desta forma, podem-se captar alguns aspectos referentes à evolução da qualidade das informações durante todo o período em que os dados são disponibilizados.

Foram utilizadas a publicação 2016 do ICIO e a publicação 2013 da WIOT, por serem baseadas na mesma classificação ISIC 3. Além disso, as publicações atuais, tanto da WIOT quanto do ICIO, abrangem períodos mais recentes, iniciando a partir de 2000 no caso da WIOT e 2005 do ICIO e a pesquisa tem como objetivo avaliar a evolução dos dados desde as publicações iniciais de cada sistema global ${ }^{1}$.

\section{Descrição do processo de construção dos sistemas utili- zados}

Os modelos globais de insumo-produto são sistemas complexos, que exigem uma grande quantidade de dados referentes ao comércio intrarregional e inter-regional. Esses dados estão, em sua maioria, disponíveis nas Tabelas de Usos e de Recursos dos países e nas bases de dados que registram o comércio internacional. Além disso, diferente dos modelos inter-regionais subnacionais, em que se tem a matriz nacional como referência, não existe uma matriz de insumo-produto mundial para ser utilizada como referência, por isso, o processo de construção é majoritariamente bottom-up em que a soma dos valores referentes a cada país resulta na matriz mundial.

\footnotetext{
${ }^{1}$ Esta pesquisa abre a possibilidade para novas comparações com publicações atuais desses sistemas globais
} 
Os dados brutos de comércio bilateral entre países têm problemas de "assimetria de fluxos". Isto é, o valor declarado pelo país A como exportação para o país B é diferente do valor declarado pelo país B como importação do país A. As fontes identificadas desses problemas de "fluxos de comércio espelho" são: (i) transbordo (transbordo é o processo pelo qual as mercadorias entram no território aduaneiro de um país, são transferidas de um meio de transporte para outro e depois deixam o mesmo porto para outro destino.); (ii) reexportação; (iii) reimportação; (iv) diferenças na valoração de preços entre CIF e FOB.

Diante dessas questões, as organizações que constroem os sistemas globais de insumo-produto podem usar diferentes estratégias no tratamento dos dados. A Tabela 1 resume o processo de construção dos dois sistemas internacionais estimados pela OCDE (ICIO) e pela WIOD (WIOT) ${ }^{2}$.

A próxima seção descreve as técnicas utilizadas para a comparação dos dois sistemas globais de insumo-produto. No entanto, as estruturas de setores e regiões do ICIO e da WIOT são diferentes, o que torna necessária, antes da comparação, uma compatibilização, que também será objeto da próxima seção.

\section{Compatibilização e Técnicas de Comparação entre o ICIO e a WIOT}

O ICIO e a WIOT utilizam a mesma classificação ISIC Rev.3 em sua construção. No entanto, a estrutura de setores e regiões é diferente em cada sistema inter-regional. Nesse contexto, antes da comparação, é preciso fazer uma compatibilização entre o ICIO e a WIOT, no intuito de reorganizá-los para que apresentem os mesmos setores e regiões. A compatibilização aqui utilizada respeita a classificação de indústrias e produtos ISIC Rev.3 e agrega os dois sistemas em 29 setores e 41 regiões comuns. No que diz respeito à WIOT, a agregação setorial mais relevante ocorre nos setores de: (i) Transporte terrestre (23); (ii) Transporte aquaviário (24); (iii) Transporte aéreo (25); e (iv) Outras atividades de transporte e agências de viagem (26); que foram agregados em um único setor. Já para o ICIO, as agregações setoriais mais relevantes foram (i) Aluguéis de máquinas e Equipamentos (27); (ii) Computadores e atividades correlatas (28); e (iii) Pesquisa e desenvolvimento e outras atividades (29); que foram agregadas em um único setor.

Em relação às regiões, a maior diferença está na região chamada "restante do mundo". O ICIO contém, originalmente, mais regiões do que a WIOT, além das regiões expandidas para o México e a China. Isso significa que vários países que estão desagregados no ICIO compõem o restante do mundo (Row) na WIOT. Logo, na agregação compativel, vários países que compõem o ICIO são agregados no restante do mundo. Para maiores detalhes da agregação ver Gonçalves Junior (2018).

\footnotetext{
${ }^{2}$ Para um entendimento detalhado do processo construção de cada sistema ver Yamano (2017) e Dietzenbacher et al. (2013)
} 
Tabela 1. STPE e WAD Intrarregional e Inter-regional para os anos de 1995 e 2011

\begin{tabular}{|c|c|}
\hline & WIOD \\
\hline $\begin{array}{l}\text { Características } \\
\text { básicas }\end{array}$ & $\begin{array}{l}\text { Edição (2013), } 40 \text { países sendo, to- } \\
\text { dos da UE mais as } 13 \text { maiores eco- } \\
\text { nomias do mundo, além do "res- } \\
\text { tante do mundo". } 59 \text { produtos e } 35 \\
\text { setores. Classificacão ISIC Rev3. }\end{array}$ \\
\hline
\end{tabular}
ICIO

Edição (2016), 63 economias, incluindo os países da OCDE, 28 que não são parte da OCDE mais o resto do mundo, além de matrizes distintas para regiões chinesas e mexicanas. 34 setores. Classificação ISIC Rev3.

Construção SUTs Nacionais
Ponto de partida são as SUTs nacionais publicadas pelos institutos de estatística dos países. Harmonização com as contas nacionais. Interpolação para os anos em que as SUTs não são publicadas utilizando dados das contas nacionais. Estimação das matrizes de Usos nacionais a preços básicos. Utilização do SUT-RAS para balanceamento.
O Ponto de partida são as contas nacionais, complementadas com fontes alternativas. As discrepâncias estatísticas existentes entre o PIB pela ótica da despesa e do produto são distribuídas para variação de estoques. O VA e o VBP por setor são estimados utilizando as matrizes insumo-produto e de produção e usos nacionais. O método de estimação das matrizes insumoproduto varia de acordo com a disponibilidade dos dados. Interpolação dos dados da matriz de produção e usos para os anos não disponiveis, balanceadas com um GRAS. Hipótese de proporcionalidade na linha para matriz de margens e importação para os anos em que não existem dados.

O total de importações e exportações deve ser o mesmo ao nível global, as diferenças são ajustadas no resto do mundo. Dados convertidos em dólares americanos a partir de estatísticas do FMI. As importações são os dados primários para a participação comercial entre parceiros. Os valores iniciais dos fluxos bilaterais para bens e serviços são separadamente estimados utilizando as participações bilaterais setoriais da BTDIxE e da EBTSI. Os valores iniciais antes do procedimento de balanceamento numérico são estimados sob a hipótese de proporcionalidade na linha para consumo intermediário e demanda final. 
Tabela 1. STPE e WAD Intrarregional e Inter-regional para os anos de 1995 e 2011 (Continuação)

\begin{tabular}{|c|c|c|}
\hline & WIOD & ICIO \\
\hline SUT interpaíses & $\begin{array}{l}\text { Assumem-se as exportações de A } \\
\text { para B como espelho das importa- } \\
\text { ções de B para A. As exportações } \\
\text { para o resto do mundo são resi- } \\
\text { duais. As estimativas iniciais de } \\
\text { consumo intermediário doméstico } \\
\text { e demanda final doméstica para o } \\
\text { resto do mundo são obtidas atra- } \\
\text { vés de médias ponderadas da parti- } \\
\text { cipação dos BRICIM e balanceadas } \\
\text { com um RAS. }\end{array}$ & $\begin{array}{l}\text { As restrições de controle dos totais } \\
\text { das linhas são os fluxos de comér- } \\
\text { cio setorial interpaíses estimados e } \\
\text { as restrições de soma nas colunas } \\
\text { são dadas pela tabela de usos im- } \\
\text { portados de cada país. A soma de } \\
\text { todo o comércio bilateral para cada } \\
\text { produto precisa ser o total impor- } \\
\text { tado do referido produto na matriz } \\
\text { de importação nacional. Utilização } \\
\text { do RAS. }\end{array}$ \\
\hline $\begin{array}{l}\text { Tabelas simétri- } \\
\text { cas }\end{array}$ & $\begin{array}{l}\text { Todo o processo inicial de constru- } \\
\text { ção é na dimensão produto x setor, } \\
\text { a conversão na dimensão setor x se- } \\
\text { tor é feita utilizando a hipótese de } \\
\text { estrutura fixa na venda dos produ- } \\
\text { tos. }\end{array}$ & $\begin{array}{l}\text { As matrizes de usos a preços bá- } \\
\text { sicos são transformadas em matri- } \\
\text { zes de insumo-produto setor x setor } \\
\text { utilizando a hipótese de estrutura } \\
\text { fixa de vendas de produtos FPSS, } \\
\text { em que a proporção para cada pro- } \\
\text { duto, dos setores que o produzem, } \\
\text { é fixa. }\end{array}$ \\
\hline
\end{tabular}

Fonte: Elaborado pelo autor.

A comparação de sistemas inter-regionais de insumo-produto pode ser feita considerando os conceitos de acurácia partitiva e holística, definidos por Jensen (1980). A acurácia partitiva foca sua atenção nos elementos das matrizes e considera a acurácia célula por célula. A acurácia holística enfatiza a interpretação de um "retrato matemático" das relações econômicas. No entanto, o termo acurácia está relacionado à existência de um valor real, utilizado como referência.

Diferente de trabalhos como os de Flegg et al. (2016) ou Tobben e Kronenberg (2015) em que existe uma matriz derivada de um censo, que serve de base para comparação, neste estudo não existe um "sistema inter-regional real" que possa servir de referência. Nesse contexto, o termo acurácia pode não ser o mais correto a ser utilizado, por isso serão empregados os termos "diferenças partitivas" e "diferenças holísticas". O objetivo principal é avaliar se os sistemas globais ICIO e WIOT são divergentes, o que significaria que a escolha entre eles pode influenciar os resultados da análise de insumo-produto.

Para avaliar o quanto os referidos sistemas globais estão próximos um do outro, em termos partitivos, serão utilizadas duas medidas que, de acordo com Lahr (2001) , são recorrentes na literatura de insumo-produto. O Standardized Total Percentage Error STPE e o Weighted Absolute Diference WAD. 
O STPE inicialmente utilizado por Leontief (1986) e posteriormente em trabalhos como os de Jalili (2000) e Lahr (2001) apresenta o erro percentual total padronizado e pode ser calculado:

$$
S T P E=100 \frac{\Sigma_{j} \Sigma_{i}\left|a_{i j}-a_{i j}^{*}\right|}{\Sigma_{j} \Sigma_{i} a_{i j}}
$$

Em que $a_{i j}$ é o i-ésimo elemento da j-ésima coluna da matriz de coeficientes técnicos A de dimensão ( $\mathrm{m} \mathrm{x} \mathrm{n)} \mathrm{utilizada} \mathrm{como} \mathrm{referência,} a_{i j}^{*}$ é o mesmo elemento da matriz de coeficientes técnicos $A^{*}$ estimada.

Para Wiebe e Lenzen (2016) , uma característica do STPE é a não simetria, isto é, o valor percentual do erro pode ser diferente dependendo da matriz que é utilizada como base. Por isso, no presente artigo, o STPE foi calculado utilizando como referência a média entre os coeficientes técnicos dos sistemas utilizados:

$$
S T P E=100 \frac{\Sigma_{j} \Sigma_{i}\left|a_{i j}-a_{i j}^{*}\right|}{\Sigma_{j} \Sigma_{i}\left[\left(a_{i j}+a_{i j}^{*}\right) / 2\right]}
$$

O WAD, utilizado em Lahr (2001), foi desenvolvido para corrigir alguns problemas de outras medidas, como a não sensibilidade a valores mais altos e a existência de zeros nas matrizes, o que torna algumas medidas indefinidas. É representado pela equação:

$$
W A D=\frac{\Sigma_{j} \Sigma_{i}\left(a_{i j}+a_{i j}^{*}\right) *\left|a_{i j}-a_{i j}^{*}\right|}{\Sigma_{j} \Sigma_{i}\left(a_{i j}+a_{i j}^{*}\right)}
$$

O termo $\left(a_{i j}+a_{i j}^{*}\right)$ pondera a diferença absoluta, de modo que os erros nos coeficientes maiores são enfatizados. Desta forma, o WAD é extremamente sensivel a erros nas células com maior valor.

A duas medidas anteriormente expostas foram utilizadas por apresentarem complementaridade entre si. O STPE não apresenta grande sensibilidade a altos valores das células comparadas, mas tem a vantagem de apresentar o erro de forma proporcional, ou seja, em porcentagem. O WAD apresenta grande sensibilidade a valores discrepantes, uma vez que a diferença entre os métodos de estimação é ponderada pelos valores dos coeficientes dos dois métodos. No entanto, como é uma medida absoluta, não apresenta resultados proporcionais, ou seja, em porcentagem.

As diferenças holísticas foram identificadas por uma análise dos (i) multiplicadores de produção; (ii) uma decomposição do valor adicionado de cada país entre a demanda final de cada país; (iii) além de uma decomposição estrutural.

Os multiplicadores de produção revelam os impactos diretos e indiretos na produção, necessários para atender a variação em uma unidade monetária na demanda 
final de cada setor em cada região. O efeito multiplicador total da produção é então obtido pelas colunas da matriz inversa de Leontief ${ }^{3}$

$$
\begin{gathered}
V A^{1}=L C V A^{11}\left(v^{11}+\cdots+v^{R 1}\right)+\cdots+L C V A^{1 R}\left(v^{1 R}+\cdots+v^{R R}\right) \\
\vdots \\
V A^{R}=L C V A^{R 1}\left(v^{11}+\cdots+v^{R 1}\right)+\cdots+L C V A^{R R}\left(v^{1 R}+\cdots+v^{R R}\right)
\end{gathered}
$$

Desta forma, pode-se calcular a proporção do Valor Adicionado de cada região $\mathrm{R}$ que está vinculada à demanda gerada por ela mesma e por cada uma das outras regiões.

Owen et al. (2014) e Owen, 2017 empregaram a decomposição estrutural para analisar as variações nos resultados obtidos a partir da utilização de sistemas interregionais de insumo-produto, construídos para as mesmas regiões, aplicando metodologias distintas. Para isso, os autores utilizaram bases de dados mundiais como WIOD; EORA e GTAP.

Para o presente estudo, a decomposição estrutural da mudança total ( $\Delta x)$ no produto bruto entre o ICIO e a WIOT pode ser caracterizada como segue:

$$
\begin{gathered}
\Delta x=0,5\left[\left(\Delta L * Y S^{I} * Y V^{I}\right)+\left(\Delta L * Y S^{W} * Y V^{W}\right)\right] \text { (Efeito tecnolgico) } \\
+0,5\left[\left(L^{W} * \Delta Y S * Y V^{I}\right)+\left(L^{I} * \Delta Y S * Y V^{W}\right)\right](\text { Efeito estrutura da demanda final) } \\
+0,5\left[\left(L^{W} * Y S^{W} * \Delta Y V\right)+\left(L^{I} * Y S^{I} * \Delta Y V\right)\right](\text { Efeito volume da demanda final })
\end{gathered}
$$

Em que

$$
\begin{gathered}
Y S^{I}=D F^{I} * i n v\left[\operatorname{diag}\left(Y V^{I}\right)\right] \\
Y S^{W}=D F^{W} * i n v\left[\operatorname{diag}\left(Y V^{W}\right)\right] \\
Y V^{I}=e^{\prime} * D F^{I} \\
Y V^{W}=e^{\prime} * D F^{W} \\
\Delta L=L^{W}-L^{I} \\
\Delta Y S=L=Y S^{W}-Y S^{I} \\
\Delta Y V=Y V^{W}-Y V^{I}
\end{gathered}
$$

Em que $L^{W}$ é a inversa de Leontief utilizando a WIOT; $D F^{W}$ é a demanda final utilizando a WIOT; $L^{I}$ é a inversa de Leontief utilizando o ICIO; $D F^{I}$ é a demanda final utilizando o ICIO e é um vetor linha de uns utilizado para a soma da demanda final nas colunas.

Diante do exposto, o método de Decomposição Estrutural permite que se identifiquem as diferenças na produção total entre os sistemas inter-regionais de insumoproduto WIOT e ICIO. Essas diferenças podem ser provenientes da tecnologia, da es-

\footnotetext{
${ }^{3}$ Maiores informações acerca do cálculo dos multiplicadores de produção, consultar (Miller e Blair, 2009)
} 
trutura da demanda final ou do volume da demanda final.

Além das diferenças holísticas e partitivas, também será a avaliada a semelhança matemática entre as matrizes estimadas por cada método. Segundo Lipschutz et al. (1994), uma matriz M é semelhante a uma matriz T se existe uma matriz não singular P tal que $M=P^{-1} T P$. Consequentemente, pode-se provar que duas matrizes semelhantes T e M apresentam as seguintes propriedades: (i) o determinante de T é igual ao determinante de $\mathrm{M}$; (ii) $\mathrm{T}$ é invertivel se, e somente se, $\mathrm{M}$ é inversível; (iii) $\mathrm{T}$ e $\mathrm{M}$ têm os mesmos autovalores, mas, em geral, não têm os mesmos autovetores; (iv) T e M têm o mesmo traço.

\section{Resultados e Discussões}

Anteriormente à verificação das diferenças holísticas e partitivas, verificou-se a semelhança matemática entre os sistemas ICIO e WIOT. Como as matrizes de coeficientes técnicos não são invertíveis, pois possuem linhas e/ou colunas linearmente correlacionadas, verificou-se a semelhança entre as matrizes inversas de Leontief estimadas por cada método. Conforme já mencionado, duas matrizes T e M são semelhantes quando possuem: (i) determinantes iguais; (ii) traços iguais; (iii) autovalores iguais.

Parar o ano de 1995, os determinantes das matrizes inversas de Leontief estimadas a partir do ICIO e da WIOT foram bastante diferentes (Det.ICIO $=1,0025 * 10^{49} \mathrm{e}$ Det. $W I O T=1,1730 * 10^{20}$ ). Os traços, por sua vez, apresentam bastante semelhança. Enquanto o traço da inversa de Leontief estimada pelo ICIO foi de 1319,30, o estimado pela WIOT foi 1305,36 , pouco mais de $1 \%$ de diferença.

No que diz respeito aos autovalores, a diferença média percentual entre os autovalores calculados a partir das inversas de Leontief estimadas pelo ICIO e pela WIOT foi de $4 \%$, com um desvio-padrão de $9 \%$. No tocante ao ano de 2011 , os determinantes foram $I C I O=1,86 * 10^{50}$ e $W I O T=2,25 * 10^{45}$. Os traços foram 1323,42 para o ICIO e 1309, 34 para a WIOT. Os autovalores apresentaram uma diferença média de 4\% e desvio-padrão de $11 \%$.

Diante do exposto, os valores anteriormente calculados apresentaram-se bastante próximos, principalmente no que tange aos traços e aos autovalores. No entanto, as matrizes inversas de Leontief estimadas pelo ICIO e pela WIOT não podem ser consideradas matematicamente semelhantes. Contudo, isso não significa que, em termos econômicos, os resultados apresentados por cada matriz não possam ser semelhantes.

As diferenças, partitiva e holística, serão avaliadas utilizando-se as matrizes de coeficientes técnicos, construídas a partir do ICIO e da WIOT para os anos de 1995 e 2011. A primeira característica a ser observada nas matrizes de coeficientes técnicos estimadas pelo ICIO e pela WIOT é se os coeficientes estimados utilizando um dos sis- 
temas inter-regionais são sistematicamente maiores ou menores do que os coeficientes estimados utilizando o outro.

Para o ano de 1995, em torno de $47 \%$ dos coeficientes estimados pelo ICIO foram menores do que os estimados pela WIOT e, para o ano de 2011 , cerca de $45 \%$. Além disso, tanto em termos setoriais quanto em termos regionais, nenhuma das matrizes estimadas apresentou algum padrão de comportamento, com os coeficientes estimados pelo ICIO sendo exclusivamente maiores/menores do que os estimados pela WIOT.

A soma de todos os elementos da matriz de coeficientes do ICIO é 604,36 para o ano de 1995 e 643,44 para o ano de 2011. Já a soma dos elementos da matriz de coeficientes técnicos da WIOT é 584,92 para 1995 e 617,78 em 2011. Isso leva a uma diferença entre os totais das matrizes de coeficientes técnicos estimadas pelos dois sistemas de 3,1\% em 1995 e 4,1\% em 2011. No entanto, as diferenças célula por célula podem assumir valores bem maiores.

No que se referem às diferenças partitivas, o STPE apresentou, para o sistema interregional como um todo, um erro percentual total padronizado de 44,6\% em 1995 e $57,2 \%$ em 2011. Para o ano de 1995, o menor STPE para os fluxos intrarregionais foi o da Itália e o maior foi o do Chipre. Já para o ano de 2011, o menor STPE intrarregional foi o da China e o maior foi o de Malta. Pode-se notar que o STPE intrarregional para o ano de 2011 é maior do que o de 1995 para 31 dos 41 países que fazem parte do sistema, o que faz com que a média do SPTE para 1995 seja de 36\% e para 2011 seja $47,3 \%$, conforme a Tabela 2 .

Em relação aos fluxos inter-regionais, os menores STPE são para a Finlândia e para a Eslováquia, tanto para 1995 quanto para 2011. Os maiores STPE inter-regionais são para Malta e Chipre, tanto para 1995 quanto para 2011. De forma geral, a média dos STPE inter-regionais estimados para todas as regiões é ligeiramente maior para 2011 do que para 1995.

Os STPE estimados para os coeficientes inter-regionais são, em média, maiores do que os intrarregionais. Isso pode ser explicado pelo fato de os dados de comércio internacional apresentarem os já mencionados problemas de "fluxo de comércio espelho". As agências que constroem o ICIO e a WIOT não lidam da mesma maneira com esses problemas e isso faz com que os fluxos de comércio estimados sejam diferentes.

Quando a distância entre os coeficientes intrarregionais e inter-regionais estimados é ponderada pelo valor dos referidos coeficientes, utilizando o WAD, os países com menor diferença absoluta ponderada são, em termos intrarregionais, Itália para 1995 e Suécia para 2011 e, em termos inter-regionais, foi Malta, tanto para 1995 quanto para 2011. As maiores diferenças intrarregionais foram para Indonésia em 1995 e Malta em 2011 e as maiores diferenças inter-regionais foram para a Rússia, tanto em 1995 quanto 2011. Conforme a Tabela 2.

De forma geral, percebe-se que, para os países da União Europeia - EU e outras 
Tabela 2. STPE e WAD Intrarregional e Inter-regional para os anos de 1995 e 2011

\begin{tabular}{|c|c|c|c|c|c|c|c|c|}
\hline \multirow[t]{2}{*}{ PAÍSES } & \multicolumn{2}{|c|}{ STPE 1995} & \multicolumn{2}{|c|}{ STPE 2011} & \multicolumn{2}{|c|}{ WAD 1995} & \multicolumn{2}{|c|}{ WAD 2011} \\
\hline & INTRA\% & INTER\% & INTRA\% & INTER\% & INTRA & INTER & INTRA & INTER \\
\hline AUS & 29,2 & 109,7 & 43 & 101,5 & 0,018 & 0,004 & 0,031 & 0,012 \\
\hline AUT & 13,8 & 72,5 & 29,2 & 76,9 & 0,007 & 0,002 & 0,014 & 0,002 \\
\hline BEL & 29,6 & 85,3 & 50,8 & 106 & 0,018 & 0,013 & 0,028 & 0,033 \\
\hline CAN & 54,9 & 90,2 & 53,7 & 104,8 & 0,031 & 0,002 & 0,038 & 0,006 \\
\hline CZE & 10,8 & 67,4 & 37,3 & 74,3 & 0,007 & 0,004 & 0,022 & 0,007 \\
\hline DNK & 24,8 & 75,6 & 69,8 & 113 & 0,012 & 0,001 & 0,045 & 0,009 \\
\hline EST & 26,5 & 70,8 & 42,1 & 88,5 & 0,013 & 0,001 & 0,024 & 0,002 \\
\hline FIN & 12 & 57,9 & 35,8 & 72,7 & 0,007 & 0,006 & 0,017 & 0,002 \\
\hline FRA & 27,4 & 67,6 & 28,3 & 83,3 & 0,016 & 0,004 & 0,018 & 0,006 \\
\hline DEU & 12,7 & 58,4 & 40,6 & 76,5 & 0,008 & 0,007 & 0,023 & 0,009 \\
\hline GRC & 53,6 & 101,1 & 55 & 111,9 & 0,026 & 0,009 & 0,024 & 0,015 \\
\hline HUN & 18,2 & 73,3 & 36,7 & 74,1 & 0,011 & 0,001 & 0,015 & 0,002 \\
\hline IRL & 65,2 & 83,7 & 61,7 & 108,5 & 0,034 & 0,001 & 0,041 & 0,001 \\
\hline ITA & 8,8 & 60,9 & 29,6 & 70,7 & 0,006 & 0,007 & 0,015 & 0,006 \\
\hline JPN & 30,4 & 82,8 & 37,9 & 80,6 & 0,019 & 0,006 & 0,033 & 0,004 \\
\hline KOR & 29,4 & 93,7 & 30,1 & 85,3 & 0,014 & 0,004 & 0,019 & 0,003 \\
\hline LUX & 54,6 & 95,7 & 81,3 & 133,1 & 0,021 & 0,001 & 0,049 & 0,003 \\
\hline MEX & 28,3 & 92,6 & 44,2 & 88 & 0,026 & 0,002 & 0,043 & 0,003 \\
\hline NLD & 25,1 & 67,7 & 40,5 & 86,4 & 0,012 & 0,004 & 0,025 & 0,009 \\
\hline POL & 36,4 & 90,2 & 25,9 & 74,6 & 0,02 & 0,003 & 0,014 & 0,003 \\
\hline PRT & 32,1 & 77,2 & 34,8 & 85,6 & 0,02 & 0,001 & 0,019 & 0,001 \\
\hline SVK & 38,2 & 58,5 & 40,3 & 61,4 & 0,026 & 0,002 & 0,022 & 0,001 \\
\hline SVN & 49,6 & 81,4 & 34,5 & 75,2 & 0,021 & 0,001 & 0,016 & 0 \\
\hline ESP & 11,9 & 68,4 & 28,8 & 78,9 & 0,008 & 0,002 & 0,018 & 0,004 \\
\hline SWE & 15,3 & 63,8 & 30,2 & 82,3 & 0,01 & 0,003 & 0,014 & 0,003 \\
\hline TUR & 48 & 102,9 & 37,4 & 87,2 & 0,028 & 0,003 & 0,028 & 0,002 \\
\hline GBR & 14,6 & 71,8 & 31,9 & 96,7 & 0,01 & 0,007 & 0,019 & 0,023 \\
\hline USA & 29,1 & 69,4 & 35,5 & 82,3 & 0,019 & 0,007 & 0,021 & 0,011 \\
\hline BGR & 46,3 & 88,5 & 61,6 & 89,1 & 0,034 & 0 & 0,038 & 0,001 \\
\hline BRA & 64,2 & 101,5 & 52,2 & 110,9 & 0,038 & 0,001 & 0,028 & 0,003 \\
\hline $\mathrm{CHN}$ & 27,4 & 107,5 & 23,8 & 82,7 & 0,026 & 0,002 & 0,021 & 0,009 \\
\hline CYP & 86,3 & 156,6 & 81,1 & 161,5 & 0,036 & 0 & 0,052 & 0,002 \\
\hline IDN & 63,8 & 96,8 & 65,1 & 97,6 & 0,05 & 0,005 & 0,046 & 0,012 \\
\hline IND & 48,7 & 107 & 43 & 107,5 & 0,038 & 0,001 & 0,025 & 0,003 \\
\hline LTU & 42,9 & 91,4 & 76,2 & 90 & 0,035 & 0,003 & 0,06 & 0,003 \\
\hline LVA & 41,3 & 78 & 54,5 & 100,7 & 0,027 & 0,001 & 0,035 & 0,003 \\
\hline MLT & 47 & 125,9 & 92,2 & 155,7 & 0,029 & 0 & 0,061 & 0 \\
\hline $\mathrm{ROU}$ & 12,9 & 93,6 & 66 & 76,9 & 0,009 & 0,001 & 0,036 & 0,001 \\
\hline RUS & 63,5 & 90,2 & 65,2 & 91,5 & 0,034 & 0,035 & 0,032 & 0,046 \\
\hline TWN & 66,1 & 77,5 & 57,5 & 86,8 & 0,035 & 0,001 & 0,041 & 0,002 \\
\hline RoW & 51,6 & 78,2 & 56,6 & 83,4 & 0,032 & 0,023 & 0,041 & 0,034 \\
\hline Média & 36,3 & 85 & 47,3 & 93,8 & 0,022 & 0,004 & 0,029 & 0,008 \\
\hline Mín. & 8,8 & 57,9 & 23,8 & 61,4 & 0,006 & 0 & 0,014 & 0 \\
\hline Máx. & 86,3 & 156,6 & 92,2 & 161,5 & 0,05 & 0,035 & 0,061 & 0,046 \\
\hline Desv. Pad. & 19 & 19,4 & 17 & 20,6 & 0,011 & 0,006 & 0,013 & 0,01 \\
\hline
\end{tabular}

Fonte: Elaborado pelo autor. 
economias mais desenvolvidas, as diferenças intrarregionais são menores do que para os outros países, pode-se inferir que isso ocorre pela qualidade de dados disponibilizados pelos institutos de estatística de cada um desses países. No que diz respeito aos fluxos inter-regionais, os dados são originários de fontes internacionais e as diferenças são provenientes de diferentes fontes utilizadas e também de como são tratados os problemas de "fluxo de comércio espelho" na construção do ICIO e da WIOT.

Os resultados do STPE e do WAD sugerem que as distâncias entre os fluxos de comércio inter-regionais, estimados pelo ICIO e pela WIOT, para os países que compõem os referidos sistemas, são maiores do que os fluxos intrarregionais. No entanto, uma análise da correlação entre os totais dos fluxos de comércio entre os países para cada setor sugere que, para a maioria dos setores, existe uma alta correlação positiva entre os fluxos inter-regionais estimados pelo ICIO e a WIOT.

A Tabela 3 apresenta os coeficientes de correlação de Pearson entre os fluxos de comércio estimados pelo ICIO e pela WIOT para cada setor, nos anos de 1995 e 2011 . Além disso, também se calculou a correlação entre todos os elementos das matrizes de comércio intermediário e final, intrarregional e inter-regional.

Os setores com maior potencial de comércio, como agricultura e indústrias, correspondem aos números 1 a 15 na Tabela 3. Os setores 16 a 29 correspondem aos serviços, com menor potencial de comércio e maior dificuldade para a obtenção de dados de comércio internacional, como (i) Educação; (ii) Saúde; (iii) Atividades imobiliárias; (iv) Serviços de alojamento e alimentação; (v) Administração pública e seguridade social; e apresentaram as menores correlações.

De forma geral, não existem grandes discrepâncias entre os coeficientes de correlação calculados para cada setor nos anos de 1995 e 2011 . A média dos coeficientes calculados para cada ano é praticamente a mesma e, para a maioria dos setores, o coeficiente calculado é bastante semelhante. As maiores diferenças estão nos setores de Telecomunicações e Administração pública.

Para esses setores e os outros setores de serviço, a disponibilidade de dados é restrita, isso abre a possibilidade para cada agência que estima os sistemas internacionais lidar com os problemas de uma forma diferente, fazendo com que as correlações sejam mais baixas.

Para a construção dos fluxos de serviços, o ICIO e a WIOT utilizam dados: (i) do EBTSI (Estimated bilateral trade in services by industry) disponibilizado pela OCDE; (ii) das Nações Unidas; e (iii) do Eurostat. No entanto, quando o dado está disponível em mais de uma fonte, a WIOT dá preferência para dados das Nações Unidas (Dietzenbacher et al., 2013)

Para Yamano (2017), a disponibilidade de estatísticas para o comércio de serviços transfronteiriços aumentou nos anos recentes. Porém, as estatísticas detalhadas não são consistentes para a maioria dos países. De acordo com Dietzenbacher et al. 
Tabela 3. Coeficientes de correlação de Pearson entre os fluxos de comércio estimados pelo ICIO e pela WIOT para cada setor, nos anos de 1995 e 2011

\begin{tabular}{|c|c|c|}
\hline Setores & 1995 & 2011 \\
\hline Toda a matriz de fluxos & 0,977 & 0,971 \\
\hline 1.Agricultura, caça, silvicultura e pesca. & 0,974 & 0,949 \\
\hline 2. Mineração e pedreiras. & 0,967 & 0,945 \\
\hline 3. Alimentos, bebidas e produtos do fumo. & 0,98 & 0,972 \\
\hline 4. Têxtil, produtos têxteis, couro e calçados. & 0,965 & 0,897 \\
\hline 5. Madeira, produtos de madeira e cortiça. & 0,977 & 0,971 \\
\hline 6. Celulose, produtos de papel, jornais e revistas. & 0,991 & 0,883 \\
\hline 7. Refino de petróleo e coque e combustível nuclear. & 0,95 & 0,94 \\
\hline 8. Química e produtos químicos. & 0,985 & 0,977 \\
\hline 9. Artigos de borracha e plástico. & 0,965 & 0,976 \\
\hline 10. Outros minerais não metálicos. & 0,958 & 0,958 \\
\hline 11. Metalurgia básica e fabricação de produtos de metal. & 0,959 & 0,941 \\
\hline 12. Outras máquinas e equipamentos não anterior. especif. & 0,987 & 0,975 \\
\hline 13. Equipamentos elétricos e ópticos. & 0,971 & 0,98 \\
\hline 14. Equipamentos de transporte. & 0,995 & 0,977 \\
\hline 15. Manufatura não ant. especificada e reciclagem. & 0,959 & 0,691 \\
\hline 16. Eletricidade e abastecimento e água e gás. & 0,701 & 0,829 \\
\hline 17. Construção civil. & 0,358 & 0,324 \\
\hline 18. Comércio de atacado, varejo e reparos. & 0,635 & 0,68 \\
\hline 19. Serviços de alojamento e alimentação. & 0,184 & 0,134 \\
\hline 20. Transporte e armazenamento. & 0,849 & 0,711 \\
\hline 21. Correios e telecomunicação. & 0,86 & 0,355 \\
\hline 22. Intermediação financeira e seguros. & 0,794 & 0,816 \\
\hline 23. Atividades imobiliárias. & 0,206 & 0,268 \\
\hline 24. Aluguél de máquinas equip. e outras ativid. comerciais. & 0,716 & 0,674 \\
\hline 25. Administração e defesa pública e securidade social. & 0,625 & 0,268 \\
\hline 26. Educação. & 0,217 & 0,44 \\
\hline 27. Saúde e assistência social. & 0,214 & 0,359 \\
\hline 28. Outros serviços comunitários, sociais e pessoais. & 0,46 & 0,465 \\
\hline 29. Atividades domésticas. & sem dados & sem dados \\
\hline Média & 0,764 & 0,727 \\
\hline Média setores serviços & 0,525 & 0,486 \\
\hline Média setores não-serviços & 0,973 & 0,938 \\
\hline Mínimo & 0,184 & 0,134 \\
\hline Máximo & 0,995 & 0,98 \\
\hline Desvio-Padrão & 0,281 & 0,275 \\
\hline
\end{tabular}

Fonte: Elaborado pelo autor. 
(2013), trabalhar com diferentes fontes tornou possivel identificar algumas inconsistências, como o fluxo de comércio registrado por uma fonte ser três vezes maior do que o registrado por outra fonte. Por isso, dependendo da fonte de dados utilizada por cada sistema (WIOT ou ICIO), os fluxos de comércio podem apresentar diferenças significativas, principalmente nos serviços.

Em seguida, será feita a análise das diferenças holísticas. A avaliação das diferenças holísticas está relacionada às diferenças entre os resultados obtidos a partir da análise de insumo-produto realizada utilizando cada um dos dois sistemas interregionais. De acordo com Jensen (1980), o objetivo mais modesto da proximidade de matrizes em termos holísticos é apropriado para matrizes regionais de insumoproduto.

Nesses termos, a proximidade entre os valores de diferentes matrizes seria julgada não pela proximidade célula por célula, mas em sua habilidade de representar o tamanho e a estrutura produtiva da economia em termos gerais. Essa interpretação implica em um elemento de "sinergia" em que a proximidade das matrizes como um todo é maior do que em seus componentes.

O primeiro elemento das diferenças holísticas a ser analisado são os multiplicadores de produção. A Figura 1 apresenta os multiplicadores de produção médios para as 41 regiões, analisadas nos anos de 1995 e 2011.

Figura 1. Multiplicadores Médios de Produção para o ICIO e a WIOT

(1)

Fonte: Elaborado pelo autor. 
De forma geral, os multiplicadores são bastante semelhantes para ambos os anos avaliados. No que diz respeito ao ano de 1995, as maiores diferenças entre os multiplicadores calculados para o ICIO e a WIOT estão na Turquia, Bulgária e Irlanda. Já para o ano de 2011, as maiores diferenças estão na Letônia, Turquia e Estônia.

Outra constatação interessante a partir da Figura 1 é que os multiplicadores de produção calculados a partir do ICIO, para a maioria dos países, tendem a ser maiores que os calculados a partir da WIOT, para o ano de 1995, apenas para seis países os multiplicadores médios, calculados a partir da WIOT, são maiores do que os calculados a partir do ICIO, já para o ano de 2011, em sete países, os multiplicadores da WIOT são maiores do que os do ICIO. No entanto, as diferenças, para a maioria dos países são relativamente pequenas e não comprometem o ranking dos multiplicadores.

Conforme já mencionado, vários indicadores de "Valor Adicionado embutido no comércio" (Trade in Value Added - TiVa) são mensurados pela OCDE, no que se refere ao comércio internacional. Diante disso, comparou-se o valor adicionado doméstico embutido na demanda final interna e externa, calculados a partir do ICIO e da WIOT, para cada um dos países que compõem o sistema. Para isso, multiplica-se o coeficiente de valor adicionado pela inversa de Leontief e, posteriormente, por uma matriz com a demanda final de cada país.

A Tabela 4 mostra algumas estatísticas das diferenças entre o valor adicionado doméstico, de cada país, embutido na demanda final dele mesmo e dos outros países. Sinais negativos indicam que o valor estimado pela WIOT é maior do que o valor estimado pelo ICIO.

As maiores diferenças positivas (ICIO>WIOT) ocorrem no Chipre, na Bélgica, na Lituânia e em Luxemburgo no ano de 1995. No ano de 2011 as maiores diferenças positivas ocorreram na Bélgica e na Holanda. Já as maiores diferenças negativas (ICIO<WIOT) ocorreram na produção do Chipre e de Malta para 1995 e do Chipre e de Luxemburgo para 2011.

Realizando uma média entre os valores de cada coluna da Tabela 4, percebe-se que a diferença máxima entre os valores estimados pelo ICIO e pela WIOT fica em média $-3,3 \%$ em 1995 e -3,8\% em 2011. Também constatou-se que não há grande variação nas diferenças entre os setores dentro de cada região, já que o desvio-padrão é menor do que 1,5\% para grande parte dos países. Além disso, de forma geral, as diferenças são baixas. Considerando todos os fluxos possiveis, nos dois anos em análise, menos de $3,0 \%$ dos valores apresentaram diferença maior do que $2 \%$, tanto positiva quanto negativa.

A análise de decomposição estrutural também pode ser utilizada para a comparação entre sistemas inter-regionais, como pode ser constatado em Owen et al. (2014) e em Owen (2017). Utilizando a decomposição estrutural, podem-se captar as diferenças existentes entre o ICIO e a WIOT ao decompor a variação total em: (i) variações tecnológicas, provenientes de diferenças na inversa de Leontief de cada sistema esti- 
Tabela 4. Estatísticas das Diferenças na Decomposição do Valor Adicionado Regional baseada na Origem da Demanda Final ICIO e WIOT

\begin{tabular}{|c|c|c|c|c|c|c|}
\hline & \multicolumn{3}{|c|}{1995} & \multicolumn{3}{|c|}{2011} \\
\hline & Máx\% & Mín\% & D-P\% & Máx\% & Mín\% & D-P\% \\
\hline AUS & 1 & $-0,7$ & 0,2 & 0,7 & $-0,8$ & 0,2 \\
\hline AUT & 3,8 & $-4,5$ & 0,9 & 2,4 & $-2,5$ & 0,6 \\
\hline BEL & 5 & $-1,4$ & 1 & 6,5 & -1 & 1,1 \\
\hline CAN & 0,8 & $-0,4$ & 0,2 & 1,2 & $-0,4$ & 0,2 \\
\hline CZE & 1,7 & $-1,7$ & 0,5 & 0,9 & -1 & 0,3 \\
\hline DNK & 0,5 & $-0,7$ & 0,2 & 1,9 & -4 & 0,7 \\
\hline EST & 3 & $-4,3$ & 1 & 3,3 & $-6,6$ & 1,2 \\
\hline FIN & 0,6 & $-1,2$ & 0,2 & 1,1 & $-1,2$ & 0,4 \\
\hline FRA & 0,4 & $-0,9$ & 0,2 & 0,4 & $-2,3$ & 0,4 \\
\hline DEU & 0,2 & $-0,2$ & 0,1 & 1,1 & $-1,5$ & 0,3 \\
\hline GRC & 2,3 & $-9,3$ & 1,6 & 1,6 & $-7,2$ & 1,3 \\
\hline HUN & 3,3 & $-7,3$ & 1,3 & 1,1 & $-3,4$ & 0,6 \\
\hline IRL & 1,5 & $-1,1$ & 0,3 & 1,5 & $-7,4$ & 1,3 \\
\hline ITA & 0,7 & $-2,7$ & 0,5 & 0,5 & $-2,2$ & 0,4 \\
\hline JPN & 0,4 & $-0,8$ & 0,1 & 0,7 & $-1,2$ & 0,3 \\
\hline KOR & 0,6 & $-1,6$ & 0,3 & 1,3 & -1 & 0,3 \\
\hline LUX & 5,2 & $-6,7$ & 1,5 & 5,6 & $-16,3$ & 3,5 \\
\hline MEX & 3 & $-1,8$ & 0,6 & 1,3 & $-0,5$ & 0,2 \\
\hline NLD & 4 & $-1,6$ & 0,7 & 6,5 & $-1,3$ & 1,1 \\
\hline $\mathrm{POL}$ & 0,4 & $-0,2$ & 0,1 & 0,8 & $-0,8$ & 0,2 \\
\hline PRT & 1,1 & $-2,3$ & 0,4 & 1,9 & $-6,9$ & 1,2 \\
\hline SVK & 0,8 & $-0,5$ & 0,2 & 0,8 & $-3,7$ & 0,6 \\
\hline SVN & 1,7 & $-4,4$ & 0,8 & 3,1 & -6 & 1,1 \\
\hline ESP & 1 & $-4,1$ & 0,7 & 1 & $-5,2$ & 0,9 \\
\hline SWE & 0,5 & $-1,3$ & 0,3 & 0,6 & $-1,7$ & 0,4 \\
\hline TUR & 2 & $-5,3$ & 0,9 & 1 & $-1,6$ & 0,3 \\
\hline GBR & 0,3 & $-1,5$ & 0,3 & 0,7 & $-1,4$ & 0,3 \\
\hline USA & 0,5 & $-0,9$ & 0,2 & 0,3 & $-1,3$ & 0,2 \\
\hline BGR & 1,5 & $-7,3$ & 1,2 & 1,6 & $-8,8$ & 1,5 \\
\hline BRA & 0,7 & $-0,5$ & 0,2 & 0,5 & $-0,9$ & 0,2 \\
\hline $\mathrm{CHN}$ & 2,3 & -1 & 0,4 & 1,2 & $-0,4$ & 0,2 \\
\hline CYP & 12,3 & -23 & 4,1 & 6,1 & $-19,8$ & 3,4 \\
\hline IDN & 2,2 & $-3,8$ & 0,7 & 0,8 & $-1,8$ & 0,3 \\
\hline IND & 1,1 & $-0,4$ & 0,2 & 2,9 & $-4,5$ & 0,8 \\
\hline LTU & 6,1 & $-2,3$ & 1,1 & 1,2 & $-6,5$ & 1,1 \\
\hline LVA & 3,8 & $-3,3$ & 0,8 & 1,5 & $-6,1$ & 1,3 \\
\hline MLT & 3,4 & $-12,7$ & 2,2 & 2,5 & $-2,8$ & 0,9 \\
\hline ROU & 0,6 & $-0,7$ & 0,2 & 1,1 & $-2,8$ & 0,6 \\
\hline RUS & 3,8 & $-4,3$ & 0,9 & 1,1 & $-3,8$ & 0,7 \\
\hline TWN & 2,3 & $-1,2$ & 0,4 & 3 & -3 & 0,7 \\
\hline RoW & 0,7 & -4 & 0,6 & 0,8 & -5 & 0,8 \\
\hline
\end{tabular}

Fonte: Elaborado pelo autor. 
mado; (ii) variações na estrutura da demanda final, resultante das diferentes formas como o valor da demanda final se distribui entre seus elementos (Consumo das famílias, Formação bruta de capital fixo, Gastos do governo, Variações de estoque e Purchase abroad by residents - CABR -; e (iii) variações no volume da demanda final, que está relacionado ao valor total de cada elemento da Demanda Final.

A Tabela 5 apresenta as variações percentuais provenientes do efeito tecnológico, da estrutura da demanda final e do volume da demanda final entre o ICIO e a WIOT para os anos de 1995 e 2011. Para realizar essa análise, utilizou-se a variação média percentual para cada país. Optou-se por trabalhar com os valores em módulo, para evitar que variações negativas anulem variações positivas. Além disso, essas variações foram ponderadas pela participação que o VBP do setor no país tem no VBP total do país, para que fosse garantida a proporcionalidade.

Para os dois anos analisados, as maiores diferenças médias estão, respectivamente, na estrutura da demanda final e na tecnologia. As diferenças tecnológicas estão relacionadas às inversas de Leontief estimadas em cada sistema. Parte das diferenças pode ser observada quando se analisaram os multiplicadores de produção, que são o resultado da soma da inversa de Leontief nas colunas. Os coeficientes inter-regionais são responsáveis pelas maiores diferenças, que, por sua vez, são provenientes das diferenças nos fluxos de comércio estimados em cada sistema.

No que diz respeito às diferenças na estrutura da demanda final, as maiores diferenças, para os dois anos analisados, estão nos valores referentes ao consumo das Instituições sem fins lucrativos (NPISH) e na Variação de Estoques (INVNT), além do Consumo no exterior pelos residentes - CABR -, esse último é zero para todos os valores no sistema da WIOT. As Tabelas 6 e 7 apresentam os valores totais dos elementos da demanda final, bem como a correlação e a diferença percentual entre eles, para os anos de 1995 e 2011.

Ao avaliar as Tabelas 6 e 7, pode-se notar que a correlação é superior a 95\% e as diferenças absolutas são inferiores a $11 \%$ para os elementos que representam a maior proporção da demanda final, como o Consumo das Famílias - HHC -; Gastos do Governo - GGFC -; e Formação Bruta de Capital Fixo - GFCF -; o que, consequentemente, resulta em alta correlação e baixa diferença absoluta quando se consideram todos os elementos da demanda final. 
Tabela 5. Diferença percentual média regional, ponderada pela participação que o VPB do setor no país tem no VBP total do país nos anos de 1995 e 2011

\begin{tabular}{|c|c|c|c|c|c|c|}
\hline \multirow[t]{2}{*}{ País } & \multicolumn{3}{|c|}{1995} & \multicolumn{3}{|c|}{2011} \\
\hline & Tecnol.\% & Estrut. DF\% & Volume DF\% & Tecnol.\% & Estrut. DF\% & Volume DF\% \\
\hline AUS & 10,6 & 10,5 & 5,3 & 13,6 & 10,3 & 6,7 \\
\hline AUT & 3,7 & 5,2 & 4,6 & 4,4 & 5,1 & 5,3 \\
\hline BEL & 4,2 & 3,9 & 4,7 & 5,2 & 5,1 & 5,3 \\
\hline CAN & 9,5 & 8,8 & 4,7 & 13,5 & 17 & 5,8 \\
\hline $\mathrm{CZE}$ & 2,6 & 6,1 & 4,7 & 6,2 & 6,8 & 6,1 \\
\hline DNK & 6,3 & 5 & 4,5 & 12,2 & 10,1 & 5,4 \\
\hline EST & 4,5 & 7,4 & 4,5 & 10 & 11,6 & 6,6 \\
\hline FIN & 4,3 & 4,6 & 4,5 & 4,2 & 6,7 & 5,3 \\
\hline FRA & 9 & 8,6 & 4,8 & 7,7 & 5,4 & 6 \\
\hline DEU & 2,7 & 2,6 & 4,8 & 6,2 & 3,4 & 6,2 \\
\hline GRC & 9,2 & 12,8 & 5,3 & 9,8 & 13,6 & 7,3 \\
\hline HUN & 6,2 & 8,3 & 4,4 & 8,4 & 8,2 & 5,2 \\
\hline IRL & 14,5 & 9,7 & 5,2 & 13,6 & 13,2 & 6,2 \\
\hline ITA & 3,5 & 2,9 & 5 & 5,9 & 4,2 & 6,3 \\
\hline JPN & 10,6 & 10,5 & 5,1 & 13 & 13,3 & 7,4 \\
\hline KOR & 8,2 & 10,3 & 5,4 & 7,2 & 7,7 & 5,2 \\
\hline LUX & 10 & 12,9 & 4,8 & 12,8 & 13,8 & 6,4 \\
\hline MEX & 7,3 & 8,3 & 3,9 & 7,7 & 7,4 & 4,2 \\
\hline NLD & 4,7 & 3,5 & 4,6 & 6,9 & 6,4 & 5,5 \\
\hline POL & 5,9 & 6,6 & 4,9 & 6,6 & 6 & 5,5 \\
\hline PRT & 6,3 & 5,2 & 4,8 & 6,6 & 5,6 & 6 \\
\hline SVK & 7 & 8,3 & 5 & 11,2 & 6,5 & 6,7 \\
\hline SVN & 6,3 & 7,8 & 4,8 & 9,5 & 8,6 & 6,2 \\
\hline ESP & 3 & 4,5 & 5,1 & 6,2 & 7,9 & 5,9 \\
\hline SWE & 4,1 & 4,1 & 4,5 & 6,1 & 6,1 & 5 \\
\hline TUR & 16,5 & 10,5 & 5,2 & 13,2 & 10,8 & 5,7 \\
\hline GBR & 4,1 & 3,8 & 4,8 & 7 & 6,8 & 5,8 \\
\hline USA & 5,9 & 14,5 & 5,1 & 8,4 & 15,1 & 6,2 \\
\hline BGR & 17,3 & 11,6 & 5,6 & 16 & 15,9 & 6,2 \\
\hline BRA & 15,7 & 15 & 4,3 & 17 & 11,2 & 5,3 \\
\hline $\mathrm{CHN}$ & 10,4 & 5,3 & 4,5 & 11,9 & 5,8 & 5,7 \\
\hline CYP & 12 & 21,9 & 4,8 & 11,2 & 12,7 & 7,7 \\
\hline IDN & 18,9 & 19 & 4,4 & 13,8 & 12,2 & 5,4 \\
\hline IND & 11,5 & 9,5 & 5,2 & 7,9 & 10,2 & 4,5 \\
\hline LTU & 10,4 & 12,8 & 4,7 & 12,8 & 20,8 & 9,4 \\
\hline LVA & 15,5 & 14,6 & 4,7 & 20,7 & 16,3 & 6,4 \\
\hline MLT & 11,3 & 10,1 & 2,2 & 11,1 & 10,7 & 2,3 \\
\hline ROU & 4 & 5,6 & 5,1 & 11,8 & 10,3 & 7,2 \\
\hline RUS & 11,2 & 12,5 & 3,4 & 13,9 & 14 & 2,4 \\
\hline TWN & 15,7 & 12,9 & 5,2 & 11,5 & 17 & 6,5 \\
\hline RoW & 13,6 & 9,6 & 4,9 & 17,3 & 11 & 5,8 \\
\hline Máx & 18,9 & 21,9 & 5,6 & 20,7 & 20,8 & 9,4 \\
\hline Max & 19 & 22 & 570 & 20,7 & 21,5 & 10,1 \\
\hline Mín & 2,6 & 2,6 & 2,2 & 4,2 & 3,4 & 2,3 \\
\hline Média & 8,7 & 9 & 4,7 & 10,3 & 10 & 5,9 \\
\hline
\end{tabular}

Fonte: Elaborado pelo autor. 
Tabela 6. Análise da estrutura e do volume dos elementos da demanda final do ICIO e da WIOT para o ano de 1995

\begin{tabular}{lcccc}
\hline \hline Elemento DF & Correlação & ICIO & WIOT & Diferença \\
\hline Todos elementos DF & 0,967 & $28.243 .897,67$ & $29.154 .246,31$ & $3,20 \%$ \\
HHC & 0,97 & $16.157 .364,10$ & $17.221 .994,95$ & $6,40 \%$ \\
NPISH & 0,197 & $350.989,15$ & $194.738,37$ & $57,30 \%$ \\
GGFC & 0,928 & $5.009 .689,28$ & $5.042 .022,78$ & $0,60 \%$ \\
GFCF & 0,989 & $6.037 .354,82$ & $6.440 .381,87$ & $6,50 \%$ \\
INVNT & 0,235 & $294.899,41$ & $255.108,35$ & $14,50 \%$ \\
CABR & Zero na WIOT & $393.600,90$ & - & - \\
\hline \hline
\end{tabular}

Fonte: Elaborado pelo autor.

Tabela 7. Análise da estrutura e do volume dos elementos da demanda final do ICIO e da WIOT para o ano de 2011

\begin{tabular}{lcccc}
\hline \hline Elemento DF & Correlação & ICIO & WIOT & Diferença \\
\hline Todos elementos DF & 0,965 & $66.643 .214,19$ & $69.263 .520,94$ & $3,90 \%$ \\
HHC & 0,969 & $36.656 .648,30$ & $39.809 .415,65$ & $8,20 \%$ \\
NPISH & 0,231 & $777.035,71$ & $384.996,05$ & $67,50 \%$ \\
GGFC & 0,936 & $12.433 .461,45$ & $12.385 .211,10$ & $0,40 \%$ \\
GFCF & 0,98 & $14.523 .967,71$ & $16.091 .263,43$ & $10,20 \%$ \\
INVNT & 0,158 & $1.258 .554,58$ & $592.634,71$ & $71,90 \%$ \\
CABR & Zero na WIOT & $993.546,45$ & - & - \\
\hline \hline
\end{tabular}

Fonte: Elaborado pelo autor.

\section{Conclusão}

O presente artigo teve como objetivo comparar os sistemas globais de insumo produto ICIO e WIOT, no intuito de identificar as principais diferenças existentes entre eles em termos holísticos e partitivos e, posteriormente, avaliar se a escolha entre os referidos sistemas pode influenciar os resultados da análise de insumo-produto.

Os dois sistemas partem das SUTs nacionais em seu processo de construção, interpolando os dados para os anos em que os países não apresentam suas SUTs. Os fluxos de comércio são obtidos junto às bases de dados internacionais EUROSTAT, Nações Unidas e OCDE. No entanto, o tratamento dispensado aos dados para o balanceamento das matrizes e para a solução de problemas de "estatísticas espelho", valoração CIF x FOB e preços básicos x preços ao consumidor são diferentes no processo de construção do ICIO e da WIOT. Isso, consequentemente, gera diferenças nos sistemas estimados. 
Em termos partitivos, os sistemas apresentaram STPE total de 45\% para 1995 e 47\% para 2011. Entretanto, trabalhos como o de Flegg et al. (2016) encontraram o melhor desempenho em comparação de sistemas regionais com STPE de 58\%, o que, em termos relativos, torna as diferenças partitivas entre o ICIO e a WIOT menores. Guando se considera região por região, a média do STPE ficou em torno de $47 \%$ para os fluxos intrarregionais e 93\% para os inter-regionais, também para o ano de 2011 , o que não sugere uma alta proximidade entre as matrizes, principalmente para os coeficientes inter-regionais.

Porém, quando se considera o WAD (que pondera as diferenças entre os coeficientes pela soma dos valores dos coeficientes em cada matriz, de modo a enfatizar diferenças em coeficientes maiores), os valores médios para os anos de 1995 e 2011 foram, respectivamente, 0,004 e 0,008 para os coeficientes inter-regionais. Isso indica que, apesar de as diferenças serem percentualmente grandes (93\%), elas são sobre valores muito baixos, o que tem pouco efeito nos resultados da análise de insumo-produto, como foi demonstrado ao avaliarem-se as diferenças holísticas.

A análise das diferenças entre os multiplicadores, sugere que, no que tange à grandeza, para algumas regiões, a diferença entre os multiplicadores de produção pode ser elevada, contudo, os rankings dos multiplicadores são bastante semelhantes quando comparados o ICIO e a WIOT, o que, em termos gerais, não compromete os resultados da análise no que diz respeito ao direcionamento de políticas.

A decomposição do valor adicionado de cada país, vinculado à demanda final doméstica e dos demais países, apresentou em média diferenças abaixo de 3,5\% entre os valores estimados pelos dois sistemas (ICIO-WIOT), tanto para 1995 quando para 2011, além disso, o desvio-padrão das diferenças entre os setores dentro de cada região foi menor que $1,5 \%$ para a maior parte das regiões.

A análise de decomposição estrutural, realizada no intuito de avaliar as diferenças tecnológicas e de estrutura e volume da demanda final, mostrou variação média relativamente baixa. As maiores variações médias apresentadas foram na tecnologia e na estrutura da demanda final (em torno de $10 \%$ para os dois anos nos dois quesitos). As diferenças na estrutura da demanda final foram principalmente devido às diferenças na variação dos estoques e no consumo das instituições sem fins lucrativos, além da CABR, que são elementos proporcionalmente menos representativos da demanda final. As diferenças tecnológicas, que são derivadas das divergências entre as inversas de Leontief estimadas pelos dois sistemas, devem-se principalmente aos valores interregionais, que por sua vez são resultado dos diferentes fluxos de comércio estimados em cada sistema.

Utilizaram-se os anos de 1995 e 2011 por serem os anos extremos das séries disponiveis, tanto para o ICIO quanto para a WIOT. Dessa forma, podem-se captar alguns aspectos referentes à evolução da qualidade das informações disponíveis durante o período analisado. As diferenças médias para o ano de 2011 foram maiores do que as 
diferenças médias para o ano de 1995. Pode-se inferir que, nos dezesseis anos entre os períodos inicial e final analisados, houve melhoria na qualidade dos dados fornecidos por vários países, especialmente os desenvolvidos. No entanto, o aumento da participação no comércio internacional de alguns países em desenvolvimento, como China, Rússia, Índia, Malta e Chipre, que segundo Yamano (2017) têm menor qualidade nos dados disponíveis, pode ter contribuído para o aumento das diferenças entre os sistemas WIOT e ICIO ocorridas no ano de 2011.

Além disso, a participação dos serviços na composição do Valor Bruto de Produção da maioria dos países aumentou no período entre 1995 e 2011 . A forma como cada sistema, WIOT e ICIO, trata os dados de serviços é diferente, isso pode fazer com que a diferença entre os dois sistemas internacionais estimados aumente.

Diante do exposto, pode-se concluir que a aparentemente alta diferença partitiva entre os valores estimados pelo ICIO e pela WIOT não se converteu em alta diferença holística, isto é, não comprometeu os resultados da análise de insumo-produto para a grande maioria dos países. Todavia, quando se trata de uma análise específica para alguns países, como Malta, Chipre, Lituânia e Luxemburgo, e/ou alguns setores, principalmente os de serviços, alguns apontamentos feitos neste estudo precisam ser considerados.

A revista Economic Systems Research publicou um número especial em 2017 tratando da criação de um Laboratório Virtual - VL - colaborativo para a compilação e operação de sistemas multirregionais de insumo-produto que utilizam larga escala de dados. De acordo com Geschke e Hadjikakou (2017), o VL possibilitará a colaboração entre um grande número de pesquisadores da área a insumo-produto, auxiliando agências estatísticas nacionais ou grupos de pesquisadores na compilação de dados para a criação de matrizes de insumo-produto.

Medidas como essa auxiliam na compilação mais eficiente das bases de dados, assegurando futuros avanços na qualidade dos dados e nas ferramentas analíticas, o que pode ajudar sobremaneira a superar os desafios ainda existentes no tratamento dos dados, principalmente de comércio internacional e, com isso, diminuir as diferenças entre os sistemas globais de insumo-produto, como o ICIO e a WIOT.

\section{Referências}

Dietzenbacher, E., Los, B., Stehrer, R., Timmer, M., e de Vries, G. (2013). The Construction of World Input output Tables in the Wiod Project. Economic Systems Research, 25(1):71-98.

Flegg, A. T., Mastronardi, L. J., e Romero, C. A. (2016). Evaluating the FLQ and AFLQ formulae for estimating regional input coefficients: empirical evidence for the province of Cordoba, Argentina. Economic Systems Research, 28(1):21-37. 
Geschke, A. e Hadjikakou, M. (2017). Virtual laboratories and MRIO analysis - an introduction. Economic Systems Research, 29(2):143-157.

Gonçalves Junior, C. A. (2018). Estimando sistemas subnacionais e globais de insumoproduto, o método é importante? comparando aplicações para o Brasil e para o mundo. Tese de Doutorado, Universidade de São Paulo.

Jalili, A. R. (2000). Comparison of Two Methods of Identifying Input-Output Coefficients for Exogenous Estimation. Economic Systems Research, 12(1):113-129.

Jensen, R. C. (1980). The concept of accuracy in regional input-output models. International Regional Science Review, 5(2):139-154.

Lahr, M. L. (2001). A Strategy for Producing Hybrid Regional Input-Output Tables, Página 211-242. Palgrave Macmillan UK.

Lenzen, M., Moran, D., Kanemoto, K., e Geschke, A. (2013). Building Eora: A Global Multi-Region Input-Output Database at High Country and Sector Resolution. Economic Systems Research, 25(1):20-49.

Leontief, W. (1986). Input-Output Economics. Oxford University Press.

Lipschutz, S., de Farias, A. A., Soares, E. F., e Júnior, A. P. (1994). Álgebra linear: teoria e problemas.

Meng, B., Zhang, Y., e Inomata, S. (2013). Compilation And Applications Of IDE-JETRO'S International Input-Output Tables. Economic Systems Research, 25(1):122-142.

Miller, R. E. e Blair, P. D. (2009). Input-output analysis: foundations and extensions. Cambridge University Press.

Moran, D. e Wood, R. (2014). Convergence between The Eora, Wiod, Exiobase, and Openeu's Consumption-Based Carbon Accounts. Economic Systems Research, 26(3):245-261.

Owen, A. (2017). Techniques for Evaluating the Differences in Multiregional InputOutput Databases. Cham: Springer International Publishing.

Owen, A., Steen-Olsen, K., Barrett, J., Wiedmann, T., e Lenzen, M. (2014). A Structural Decomposition Approach to Comparing MRIO Databases. Economic Systems Research, 26(3):262-283.

Stadler, K., Steen-Olsen, K., e Wood, R. (2014). The "Rest of the World" - Estimating the Economic Structure of Missing Regions in Global Multi-Regional Input-Output Tables. Economic Systems Research, 26(3):303-326.

Steen-Olsen, K., Owen, A., Hertwich, E. G., e Lenzen, M. (2014). Effects of Sector Aggregation on $\mathrm{CO}_{2}$ Multipliers in Multiregional Input-Output Analyses. Economic Systems Research, 26(3):284-302. 
Tobben, J. e Kronenberg, T. H. (2015). Construction of Multi-Regional Input-Output Tables Using the Charm Method. Economic Systems Research, 27(4):487-507.

Tukker, A. e Dietzenbacher, E. (2013). Global Multiregional Input-Output Frameworks: An Introduction and Outlook. Economic Systems Research, 25(1):1-19.

Wiebe, K. S. e Lenzen, M. (2016). To RAS or not to RAS? What is the Difference in Outcomes in Multi-Regional Input-Output Models? Economic Systems Research, 28(3):383-402.

Yamano, N. (2017). Development of Global Inter-Country Inter-Industry System for Various Policy Perspectives. Tese de Doutorado, University of Illinois at UrbanaChampaign.

(๔) Este artigo está licenciado com uma CC BY 4.0 license. 